\title{
Downlink Transmission of Broadband OFCDM Systems_-Part I: Hybrid Detection
}

\author{
Yiqing Zhou, Member, IEEE, Jiangzhou Wang, Senior Member, IEEE, and Mamoru Sawahashi, Member, IEEE
}

\begin{abstract}
The broadband orthogonal frequency and code division multiplexing (OFCDM) system with two-dimensional spreading (time and frequency domain spreading) is becoming a very attractive technique for high-rate data transmission in future wireless communication systems. In this paper, a quasianalytical study is presented on the downlink performance of the OFCDM system with hybrid multi-code interference (MCI) cancellation and minimum mean square error (MMSE) detection. The weights of MMSE are derived and updated stage by stage of MCI cancellation. The effects of channel estimation errors and sub-carrier correlation are also studied. It is shown that the hybrid detection scheme performs much better than pure MMSE when good channel estimation is guaranteed. The power ratio between the pilot channel and all data channels should be set to 0.25 , which is a near optimum value for the two-dimensional spreading system with time domain spreading factor $\left(N_{T}\right)$ of 4 and 8 . On the other hand, in a slow fading channel, a large value of the channel estimation window size $\gamma N_{T}$, where $\gamma$ is an odd integer, is expected. However, $\gamma=3$ is large enough for the system with $N_{T}=8$ while $\gamma=5$ is more desirable for the system with $N_{T}=4$. Although performance of the hybrid detection degrades in the presence of the sub-carrier correlation, the hybrid detection still works well even the correlation coefficient is as high as 0.7 . Finally, given $N_{T}$, although performance improves when the frequency domain spreading factor $\left(N_{F}\right)$ increases, the frequency diversity gain is almost saturated for a large value of $N_{F}$ (i.e., $N_{F} \geq 32$ ).
\end{abstract}

Index Terms-OFDM, two-dimensional spreading, multicarrier CDMA, interference cancellation, MMSE detection, correlated fading channels, channel estimation.

\section{INTRODUCTION}

$\mathbf{U}$ SING a huge bandwidth, future wireless systems aim to achieve data rates as large as $100 \mathrm{Mbps}$, especially in the downlink. With such a wide bandwidth, a transmit signal experiences a broadband channel revealing many multipaths, which may cause severe multipath interference. It has been shown in [1]-[5] that orthogonal frequency and code division multiplexing (OFCDM), or orthogonal multi-carrier code division multiple access (MC-CDMA), exhibits better performance than conventional direct sequence CDMA (DS-CDMA) scheme in the broadband channel. This is because OFCDM is

Paper approved by N. Al-Dhahir, the Editor for Space-Time, OFDM, and Equalization of the IEEE Communications Society. Manuscript received October 15, 2003; revised March 6, 2004 and July 2, 2004. This paper was presented at VTC Spring 2004, Milan, Italy, May 2004.

Y. Zhou and J. Wang are with Department of Electrical and Electronic Engineering, University of Hong Kong, Hong Kong (e-mail: yqzhou@eee.hku.hk; jwang@eee.hku.hk).

M. Sawahashi is with IP Radio Network Development Department, NTT DoCoMo, Inc., Yokosuka-shi, Kanagawa 239-8536, Japan (e-mail: sawahasi@ mlab.yrp.nttdocomo.co.jp)

Digital Object Identifier 10.1109/TCOMM.2005.844962 based on conventional orthogonal frequency division multiplexing (OFDM), and employs a large number of orthogonal sub-carriers to transmit symbols in parallel, so that the symbol duration is increased substantially and the system can combat the multipath interference. In order to have frequency diversity, the same data in OFCDM modulates a number of sub-carriers in terms of frequency domain spreading. Since time domain spreading is also introduced, multiple codes in both domains can be used to transmit high rate data with high efficiency. In the OFCDM systems with both time and frequency domain spreading, the total spreading factor $(N)$ is the product of time domain spreading factor $\left(N_{T}\right)$ and frequency domain spreading factor $\left(N_{F}\right)$, i.e., $N=N_{T} \times N_{F}$.

Multi-code transmission is one of efficient ways to achieve high-speed transmission [6]. The multi-code channels are orthogonal in either time domain or frequency domain in a Gaussian or flat fading channel. However, in a realistic mobile channel, the orthogonality no longer maintains in time domain because of possible fast fading or in frequency domain because of different fading among sub-carriers. Since a short packet length (e.g., $0.5 \mathrm{~ms}$ ) is preferred in high-speed packet transmission, the channel variation in one packet duration is negligible in most cases. Hence, time domain spreading can preserve the orthogonality between code channels. On the other hand, since the broadband channel is highly frequency selective, the orthogonality between code channels in frequency domain will be distorted by the frequency selectivity, so that multi-code interference (MCI) may occur. In other words, even though the system performance may be improved by exploiting frequency diversity, MCI is caused. In order to improve performance, MCI must be cancelled out as much as possible. Thus, it is desirable to evaluate the performance of the OFCDM system when MCI cancellation technique is adopted.

The OFCDM system with only frequency domain spreading has been well studied in [7]-[11]. Yee and Linnartz firstly introduced OFCDM concept in [7], where simple combining (or detection) schemes are also proposed with ideal channel state information, such as equal gain combining (EGC) and maximum ratio combining (MRC). By means of computer simulation, [8] compared the performance of different detection techniques, including EGC, MRC, zero-forcing, controlled equalization, minimum mean square error (MMSE), maximum likelihood detection (MLD) and iterative detection. MLD is optimum but its complexity increases exponentially with the number of code channels. Traditional MMSE is effective but complex. However, it can be simplified in case of full load and slow fading channels [9]. The simplified MMSE has been widely studied in [9], [10]. However, so far interference cancellation technique has not been 


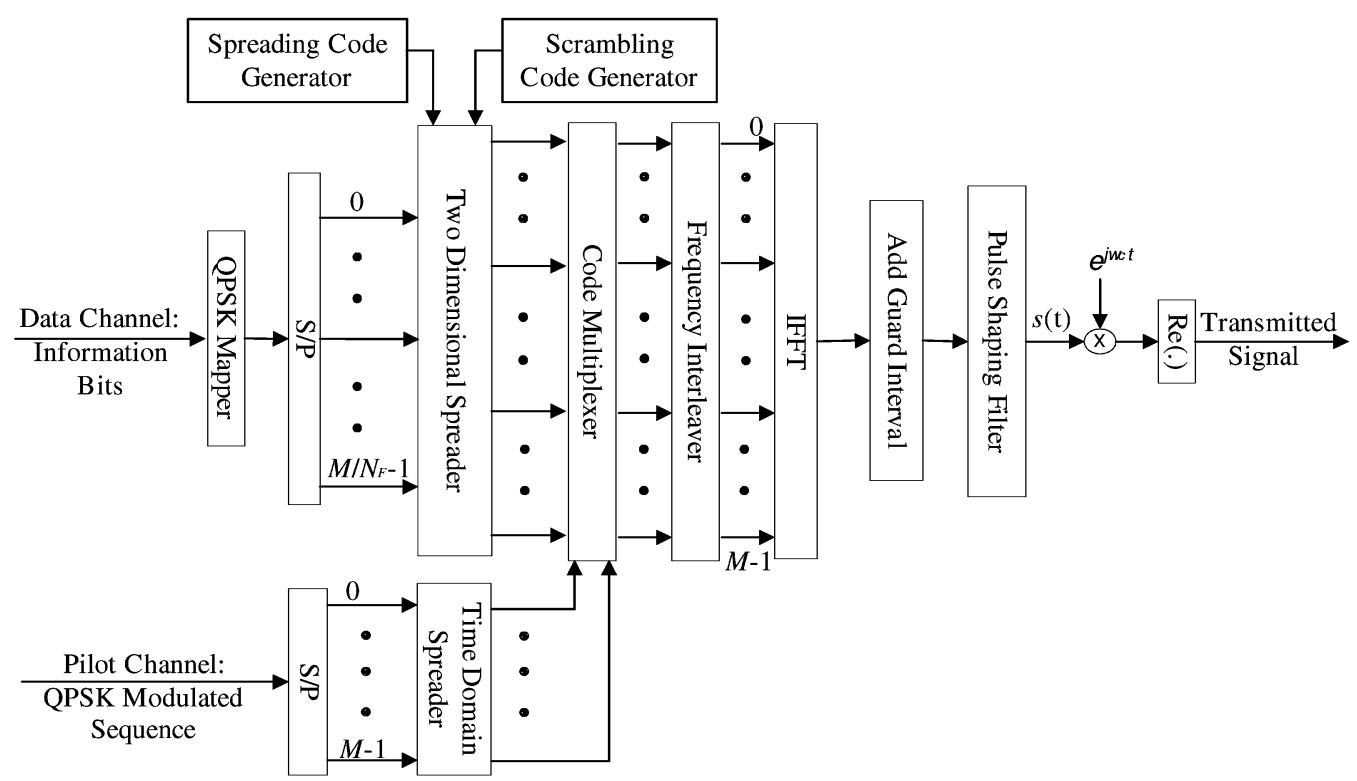

Fig. 1. Transmitter structure of the OFCDM system.

investigated yet for the OFCDM system. The objective of this paper is to analytically investigate the performance of OFCDM with hybrid MCI cancellation and MMSE detection.

The rest of the paper is organized as follows. Section II introduces the system model, including structures of transmitter and receiver. Section III is devoted to the study of decision variables of OFCDM receiver. Pure MMSE detection and hybrid MCI cancellation and MMSE detection are presented in detail. The error probability is discussed in Section IV. Section V presents some representative numerical results. Finally, Section VI draws conclusion.

\section{SYSTEM MODEL}

\section{A. System Description}

The transmitter block diagram of the OFCDM system is shown in Fig. 1. For each data channel, 0- or 1-valued information bits are firstly processed by a QPSK mapper with Gray coding. Suppose the OFCDM system employs $M$ sub-carriers. Since the frequency domain spreading factor is $N_{F}$, totally $N_{B}=M / N_{F}$ QPSK symbols can be spread in frequency domain at the same time. Therefore, the output of QPSK mapper is serial-to-parallel ( $\mathrm{S} / \mathrm{P})$ converted into $N_{B}$ streams. Every symbol of the streams will be two-dimensionally spread with $N_{T}$ chips in time domain and $N_{F}$ chips in frequency domain. At the same time, known QPSK modulated pilot symbols are $\mathrm{S} / \mathrm{P}$ converted into $M$ streams. Each pilot symbol of the streams is spread only with $N_{T}$ chips in time domain. Then all code channels are combined at code multiplexer. After code multiplexing, the combined signal is scrambled. A frequency interleaver is then employed to separate the $N_{F}$ sub-carriers carrying the same symbol as far as possible, so that the system can benefit from frequency diversity due to independent fading. After interleaving, totally $M$ chips should be up-converted to $M$ sub-carriers and transmitted in parallel. An $M$-point IFFT realizes this operation. At the output of IFFT, an effective OFCDM symbol with duration $T_{e}$ is obtained with $M$ samples.
A guard interval with $L_{g}$ samples (or duration $T_{g}$ ) is inserted between OFCDM symbols to prevent intersymbol interference. Hence, a complete OFCDM symbol is composed by $M+L_{g}$ samples with duration $T=T_{e}+T_{g}$. Finally, OFCDM symbols pass through pulse shaping filter, are up-converted to carrier frequency and transmitted.

At the receiver (see Fig. 2), signals are firstly down-converted to baseband signals. With recovered timing information, signals are processed by a matched filter where the guard interval is removed. The resultant signals further input to FFT block, which realizes the $M$-sub-carrier down conversion. After FFT, the $M$ chips involved with $M$ sub-carriers are obtained, deinterleaved and descrambled. On one hand, the output of the descrambler is accumulated to carry out channel estimation. Since the all "1" time domain spreading code is assigned to the pilot channel, the channel estimator is simply realized by the summation in time domain. The estimated channel fading will be used to generate the MMSE weights. On the other hand, the output of the descrambler is passed to the time domain despreader for data channels. When the MCI cancellor is employed, the output from the time domain despreader will be subtracted by regenerated MCI. Note that the regenerated MCI is unavailable at the zeroth stage (or original stage) of MCI cancellation, because no recovered data are available. Therefore, at the zeroth stage the regenerated MCI is simply set to 0 . The remainders are then multiplied by weights obtained from MMSE algorithms and combined at the frequency domain despreader to get the signals of a desired code channel. After despreading in frequency domain, a hard decision is used to recover QPSK symbols. Then signals are parallel-to-serial (P/S) converted and demapped. Finally, information bits at the desired code channel are obtained, which will be used to regenerate the MCI for other data channels. Similarly, the recovered information bits from other data channels are also used to regenerate the MCI for the current data channel. Basically, the interference regenerator performs the operation as the transmitter except channel information. Although MCI cancellation cannot be performed at the zeroth stage due 


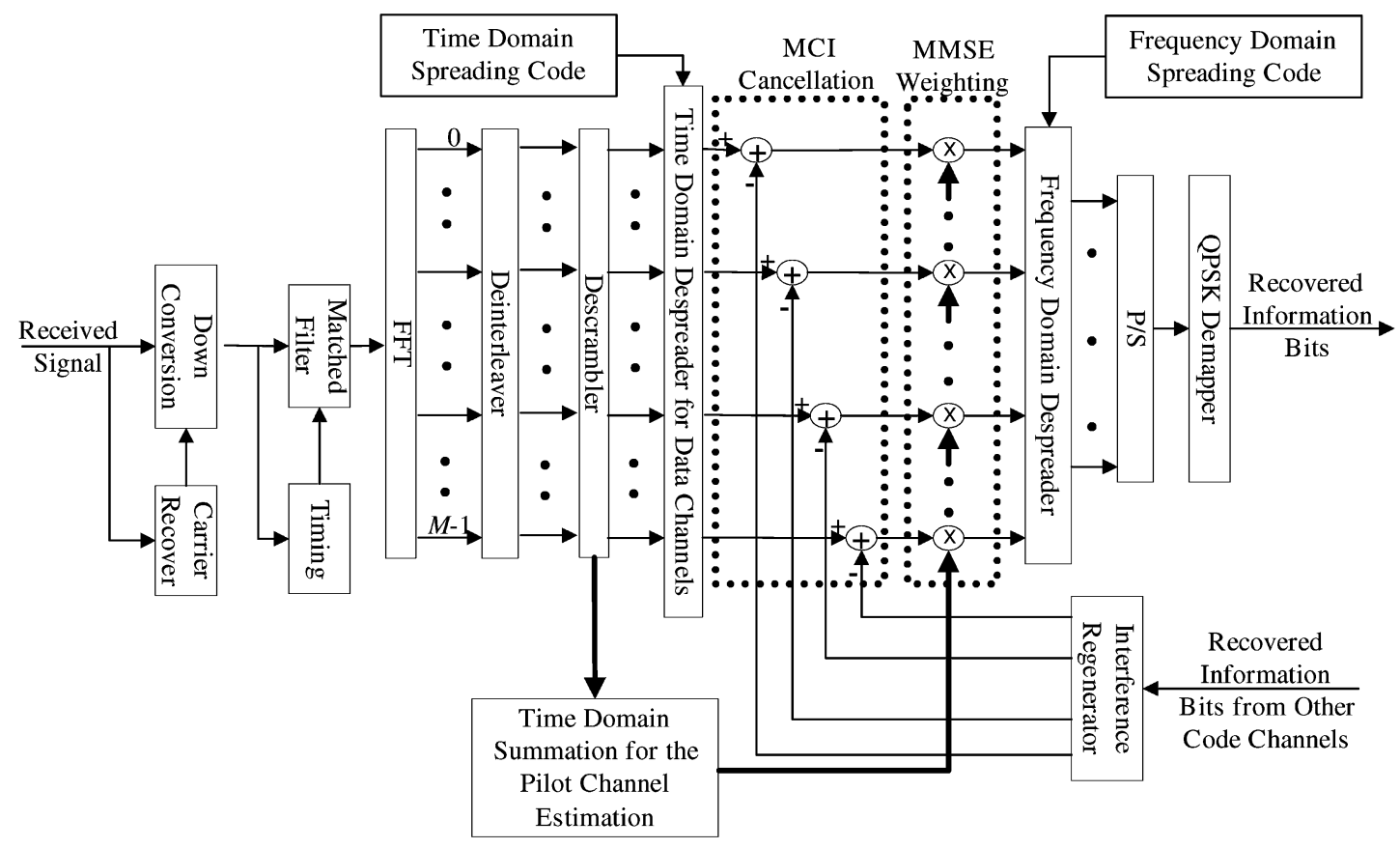

Fig. 2. Receiver structure for OFCDM with hybrid MCI cancellation and MMSE detection.

to unavailable information data, at the first stage, the signals at the output of the time domain despreader will be subtracted by nonzero MCI. This cancellation process will continue in an iterative way until the specified number of stages is reached. Since the MMSE weights are related to the input power, they must be updated stage by stage due to the reduction of the MCI in each stage. Generally speaking, as the recovered information bits become more reliable stage by stage, MCI can be regenerated with higher accuracy stage by stage. After subtraction, MCI can be cancelled out much from the received signal. Thus the bit error rate (BER) performance can be improved stage by stage.

\section{B. Two-Dimensional Spreading Sequences}

As defined before, the total spreading factor of the two-dimensional spreading is expressed as $N=N_{T} \times N_{F}$. The QPSK symbol transmitted on the $k$ th $(k=0, \ldots, K-1)$ code channel is spread by a one-dimensional code with length $N$, denoted as $C^{(k)}=\left\{c_{0}^{(k)}, \ldots, c_{N-1}^{(k)}\right\}$. The $N$ chips of the code consist of $N_{T}$ chips per sub-carrier over $N_{F}$ sub-carriers as shown in Fig. 3. With frequency interleaving, the first $N_{T}$ chips, $c_{0}^{(k)}, \ldots, c_{N_{T}-1}^{(k)}$, are impressed on the zeroth sub-carrier, the second $N_{T}$ chips, $c_{N_{T}}^{(k)}, \ldots, c_{2 N_{T}-1}^{(k)}$, are impressed on the $\left(M / N_{F}\right)$ th sub-carrier, and so on. Note that all code chips take 1 or -1 . When orthogonal VSF (OVSF) code is chosen as the spreading code, a corresponding time domain OVSF spreading code of length $N_{T}$, i.e., $C_{N_{T}}^{\left(k_{T}\right)}=\left\{c_{N_{T}, 0}^{\left(k_{T}\right)}, \ldots, c_{N_{T}, N_{T}-1}^{\left(k_{T}\right)}\right\}$, can be derived with a corresponding frequency domain OVSF spreading code of length $N_{F}$, i.e., $C_{N_{F}}^{\left(k_{F}\right)}=$ $\left\{c_{N_{F}, 0}^{\left(k_{F}\right)}, \ldots, c_{N_{F}, N_{F}-1}^{\left(k_{F}\right)}\right\}$. The relationship among $C^{(k)}, C_{N_{T}}^{\left(k_{T}\right)}$,

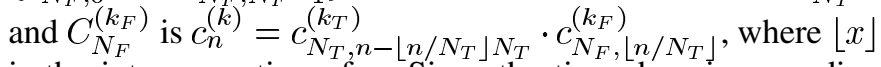
is the integer portion of $x$. Since the time domain spreading code $C_{N_{T}}^{(0)}$ is reserved for the pilot channel, there are only $N_{T}-1$ time domain spreading codes available for data channels. Hence, the relationship among $k, k_{T}$ and $k_{F}$ is $k_{T}=k-\left\lfloor k /\left(N_{T}-1\right)\right\rfloor\left(N_{T}-1\right)+1, k_{T}=1, \ldots, N_{T}-1$, and $k_{F}=\left\lfloor k /\left(N_{T}-1\right)\right\rfloor, k_{F}=0,1, \ldots, N_{F}-1$. Equivalently, the $k$ th code channel employs a two-dimensional spreading code $\left\{C_{N_{T}}^{\left(k_{T}\right)}, C_{N_{F}}^{\left(k_{F}\right)}\right\}$. Assuming that $K$ is the total number of code channels, for the $k$ th code channel, the code set of other $K-1$ code channels can be divided into two sub-sets: one set, $\Omega_{F}$, with the same time domain spreading code as the $k$ th code but different frequency domain spreading codes, and the other set, $\Omega_{T}$, with different time domain spreading codes, given respectively by

$$
\begin{aligned}
\Omega_{F} & =\left\{C_{N_{T}}^{\left(k_{T}\right)} C_{N_{F}}^{\left(\bar{k}_{F}\right)}\right\} \\
\bar{k}_{F} & =0, \ldots, N_{F}-1 \quad \text { but } \bar{k}_{F} \neq k_{F} \\
\Omega_{T} & =\left\{C_{N_{T}}^{\left(\bar{k}_{T}\right)}, C_{N_{F}}^{\left(\bar{k}_{F}\right)}\right\} \quad \bar{k}_{F}=0, \ldots, N_{F}-1 \\
\bar{k}_{T} & =1, \ldots, N_{T}-1 \quad \text { but } \bar{k}_{T} \neq k_{T} \neq 0
\end{aligned}
$$

According to the orthogonality of codes in frequency or time domain spreading, one obtains

$$
\sum_{i=0}^{N_{F}-1} c_{N_{F}, i}^{\left(k_{F}\right)} c_{N_{F}, i}^{\left(\bar{k}_{F}\right)}=0 \quad k_{F} \neq \bar{k}_{F}
$$

and

$$
\sum_{i=0}^{N_{T}-1} c_{N_{T}, i}^{\left(k_{T}\right)} c_{N_{T}, i}^{\left(\bar{k}_{T}\right)}=0 \quad k_{T} \neq \bar{k}_{T} .
$$

Code channels in different sub-sets $\left(\Omega_{F}\right.$ or $\left.\Omega_{T}\right)$ have different contribution to the MCI on the $k$ th code channel. In highly frequency selective channels, code channels from $\Omega_{F}$ cause severe MCI to the $k$ th code channel because their orthogonality in frequency domain is distorted by frequency selective fading on sub-carriers. On the other hand, in a slow fading channel or in a short packet, the orthogonality in time domain between 


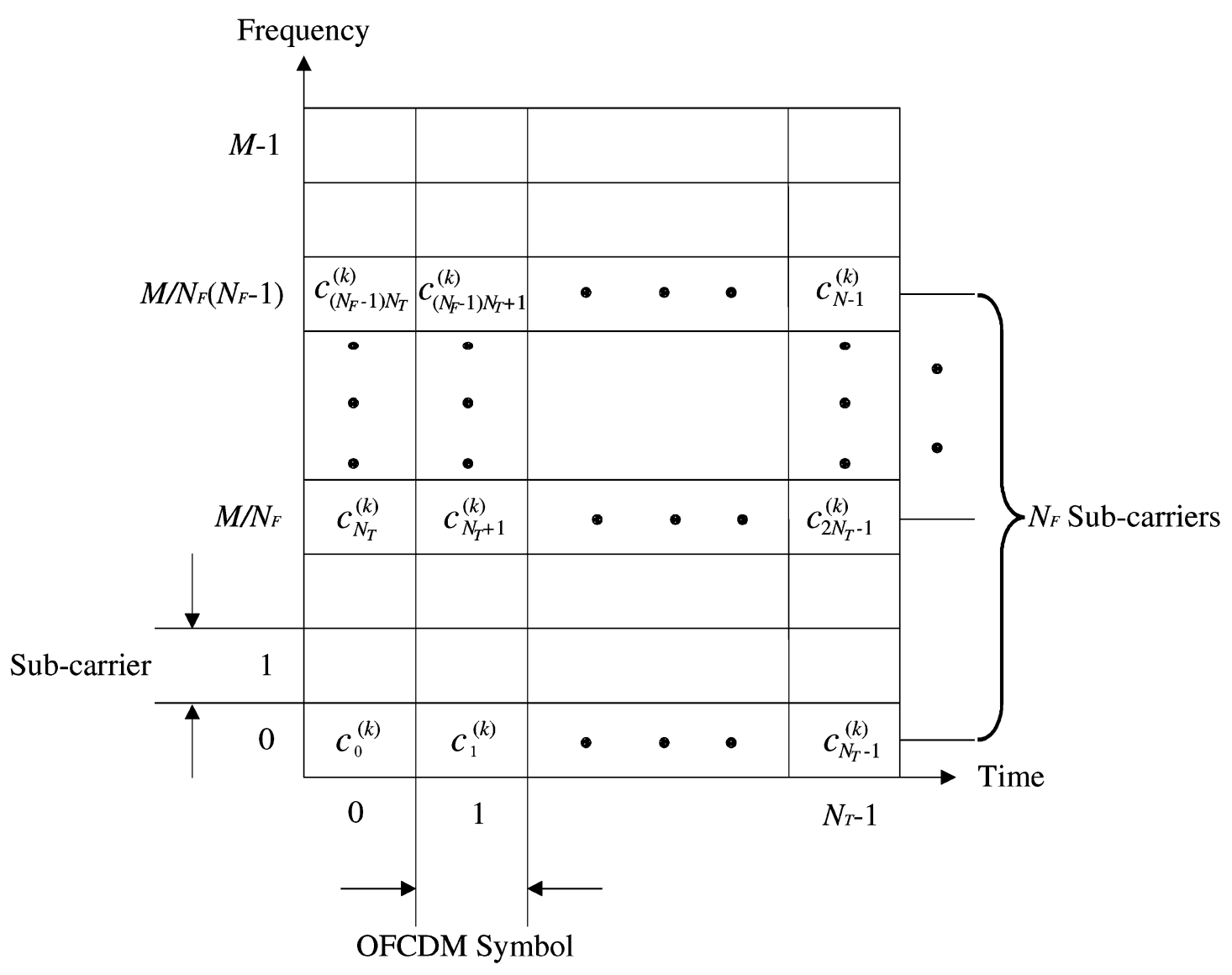

Fig. 3. Two-dimensional spreading in both time and spreading domains.

any code channel from $\Omega_{T}$ and the $k$ th code channel is maintained. Thus, there is no MCI from $\Omega_{T}$. Therefore, only code channels from $\Omega_{F}$ cause MCI to the $k$ th code channel. The number of effective interference code channels is equal to the number of code channels in $\Omega_{F}$, which is defined as $K_{C}$. As there are totally $N_{T}-1$ time domain orthogonal codes for data channels, when $K$ is less than or equal to $N_{T}-1$, orthogonality between any two code channels can be maintained by assigning channels with different time domain orthogonal codes. In this case, $\Omega_{F}$ does not exist. However, when $K$ is larger than $N_{T}-1$, reuse of the same time domain spreading codes is unavoidable and $\Omega_{F}$ must exist, so that MCI occurs. To keep MCI small for each code channel, time domain spreading codes should be assigned first and the code assignment will be carried out as follows: $\left\{C_{N_{T}}^{(1)}, C_{N_{F}}^{(0)}\right\}$ for the zeroth code channel, $\left\{C_{N_{T}}^{(2)}, C_{N_{F}}^{(0)}\right\}$ for the first channel, $\ldots,\left\{C_{N_{T}}^{\left(N_{T}-1\right)}, C_{N_{F}}^{(0)}\right\}$ for the $\left(N_{T}-2\right)$ th channel, $\left\{C_{N_{T}}^{(1)}, C_{N_{F}}^{(1)}\right\}$ for the $\left(N_{T}-1\right)$ th channel, and so on. Note that according to this code assignment method, the number, $K_{C}$, of interfering code channels in $\Omega_{F}$ may not be the same for each code channel, and $K_{C}$ will be at most

$$
K_{C}=\left\lfloor(K-1) /\left(N_{T}-1\right)\right\rfloor \text {. }
$$

\section{DECISION VARIABLES}

\section{A. Transmitted Signal and Channel Model}

As described above, there are $N_{B}=M / N_{F}$ QPSK symbols spread in frequency domain at the same time. Consider the zeroth symbol. With block frequency interleaving, it is impressed on the zeroth, $\left(N_{B}\right)$ th, $\ldots,\left(\left(N_{F}-1\right) N_{B}\right)$ th sub-carriers. Therefore, in downlink, the transmitted zeroth data signal of the $m$ th sub-carrier $\left(m=0, N_{B}, \ldots,\left(N_{F}-1\right) N_{B}\right)$ on the $i$ th OFCDM symbol $\left(i=0, \ldots, N_{T}-1\right)$ is expressed as

$$
S_{m, i}(t)=\sqrt{P} \sum_{k=0}^{K-1} a_{k, m, i} e^{j 2 \pi f_{m}(t-i T)} p(t-i T)
$$

where $P$ is the signal power of one data code on one sub-carrier, and $a_{k, m, i}$ is given by

$$
a_{k, m, i}=d_{k} c_{N_{T}, i}^{\left(k_{T}\right)} c_{N_{F}, m / N_{B}}^{\left(k_{F}\right)} s_{m, i}
$$

where $d_{k}$ is the data symbol of the $k$ th code channel, $s_{m, i}$ is the $i$ th chip on the $m$ th sub-carrier of the random binary scrambling code, and $f_{m}=m / T_{e}$ is the baseband equivalent frequency of the $m$ th sub-carrier. $p(t)$ is assumed rectangular as

$$
p(t)= \begin{cases}1, & -T_{g}<t \leq T_{e} \\ 0, & \text { elsewhere. }\end{cases}
$$

In order to carry out effective channel estimation, a code-multiplexed pilot channel is employed on each sub-carrier. Pilot symbols are spread only in time domain with $C_{N_{T}}^{(0)}$. The baseband pilot signal of the $m$ th sub-carrier on the $i$ th OFCDM symbol can be expressed as

$$
S_{P, m, i}(t)=\sqrt{\beta P} \cdot a_{P, m, i} e^{j 2 \pi f_{m}(t-i T)} p(t-i T)
$$


where $\beta$ is the power ratio of pilot to one data channel and

$$
a_{P, m, i}=d_{P} c_{N_{T}, i}^{(0)} s_{m, i}=d_{P} s_{m, i}
$$

where $d_{P}$ is the known pilot symbol and $c_{N_{T}, i}^{(0)}=1$ for all $i$ of the pilot code $C_{N_{T}}^{(0)}$. Therefore, the total baseband equivalent complex transmitted signal in $N_{T}$-OFCDM-symbol duration is given by

$$
S(t)=\sum_{m=0}^{M-1} \sum_{i=0}^{N_{T}-1}\left[S_{m, i}(t)+S_{P, m, i}(t)\right] .
$$

Although the broadband channel is highly frequency selective, the signal transmitted on each sub-carrier experiences a flat fading channel. Suppose the fading rate is slow and the fading is approximately fixed over $\gamma N_{T}$ OFCDM symbols, where $\gamma$ is an odd integer. Hence, $h_{m}$ is used to denote the complex channel fading for the $m$ th sub-carrier over $\gamma N_{T}$ OFCDM symbols. The amplitude and phase of $h_{m}$ are assumed to be Rayleigh distributed with $E\left\{\left|h_{m}\right|^{2}\right\}=1$ and uniformly distributed in $[0,2 \pi]$, respectively. Furthermore, the correlation coefficient between $h_{m}$ and $h_{\bar{m}}$ is given by [12]

$$
\rho_{m, \bar{m}}=E\left\{h_{m}^{*} h_{\bar{m}}\right\}=\frac{1}{\left.\sqrt{1+\left(\Delta f / \Delta f_{c}\right.}\right)^{2}}
$$

where $(\cdot)^{*}$ stands for the conjugate operation, $\Delta f$ is the frequency separation between the $m$ th and $\bar{m}$ th sub-carriers, and $\Delta f_{c}$ is the coherence bandwidth of the channel. Although a huge bandwidth, e.g., $100 \mathrm{MHz}$, is considered for the OFCDM system and frequency interleaving is employed, the correlation between the $N_{F}$ sub-carriers carrying the same data can still be large for a large $N_{F}$. Since this correlation has a direct effect on the value of MCI and frequency diversity gain, it is necessary to study the system performance in the presence of correlation among sub-carriers.

\section{B. Received Signal and Channel Estimation}

The received signal at the $\bar{m}$ th $\left(\bar{m}=0, N_{B}, \ldots,\left(N_{F}-\right.\right.$ 1) $N_{B}$ ) sub-carrier on the $\bar{i}$ th OFCDM symbol is given by

$$
r_{\bar{m}, \bar{i}}(t)=h_{\bar{m}}\left[S_{\bar{m}, \bar{i}}(t)+S_{P, \bar{m}, \bar{i}}(t)\right]+\eta_{\bar{m}, \bar{i}}(t)
$$

where $\eta_{\bar{m}, \bar{i}}(t)$ is a complex additive white Gaussian noise (AWGN) on the $\bar{m}$ th sub-carrier with power spectrum density of $N_{0}$. Both $h_{\bar{m}}$ and $\eta_{\bar{m}, \bar{i}}(t)$ are assumed to be independent. With ideal symbol timing, this baseband signal inputs to the matched filter. By setting $T_{g}$ larger than the maximum channel delay, there is no interference from adjacent OFCDM symbols. Then the output of the matched filter for the $\bar{m}$ th sub-carrier on the $\bar{i}$ th OFCDM symbol is given by

$$
\begin{aligned}
r(\bar{m}, \bar{i})= & \frac{1}{T_{e}} \int_{\bar{i} T}^{\bar{i} T+T_{e}} r_{\bar{m}, \bar{i}}(t) e^{-j 2 \pi f_{\bar{m}}(t-\bar{i} T)} \\
& \cdot p\left(T_{s}-(t-\bar{i} T)\right) d t \\
= & h_{\bar{m}} \sqrt{P} \sum_{k=0}^{K-1} a_{k, \bar{m}, \bar{i}}+h_{\bar{m}} \sqrt{\beta P} a_{P, m, \bar{i}}+N(\bar{m}, \bar{i})
\end{aligned}
$$

which contains three terms: the first two terms stand for the data and pilot signal, respectively, and the third term is background noise with variance

$$
\sigma_{n}^{2}=E\left\{|N(\bar{m}, \bar{i})|^{2}\right\}=\frac{N_{0}}{T_{e}} .
$$

In order to demodulate the data signal, the channel estimation is needed. Since the all "1" code is assigned to the pilot channel, the output of the matched filter is descrambled and input to the time domain summation for the pilot channel to carry out channel estimation. Actually, the summation is to take simple average of the output of the descrambler over $\gamma N_{T}$-OFCDMsymbol duration (the channel fading is assumed to be constant in these $\gamma N_{T}$-OFCDM-symbol duration). Therefore, the resultant output of the summation for pilot is given by

$$
\begin{aligned}
r_{P}(\bar{m}) & =\frac{1}{\gamma N_{T}} \sum_{\bar{i}=-(\gamma-1) N_{T} / 2}^{(\gamma+1) N_{T} / 2-1} r(\bar{m}, \bar{i}) s_{\bar{m}, \bar{i}} \\
& =\sqrt{\beta P} d_{P} h_{\bar{m}}+N_{P}
\end{aligned}
$$

where the first term is the useful component. Notice that there is no interference in (16) from data channels because of the orthogonality of time domain spreading codes. $N_{P}$ is the noise term with variance

$$
\sigma_{N_{P}}^{2}=\frac{\sigma_{n}^{2}}{\gamma N_{T}}=\frac{N_{0}}{\gamma N_{T} T_{e}} .
$$

Therefore, the channel estimation for $N_{T}$ OFCDM symbols $\left(\bar{i}=0,1, \ldots, N_{T}-1\right)$ is given by

$$
\hat{h}_{\bar{m}}=\frac{r_{P}(\bar{m})}{\sqrt{\beta P} d_{P}}=h_{\bar{m}}+e_{P, \bar{m}}
$$

where $e_{P, \bar{m}}$ is the channel estimation error with variance

$$
\sigma_{e_{P, \bar{m}}}^{2}=\frac{\sigma_{N_{P}}^{2}}{\beta P}=\frac{N_{0}}{\gamma N_{T} T_{e} \beta P} .
$$

It is assumed that the zeroth data code with two-dimensional spreading code $\left\{C_{N_{T}}^{(1)}, C_{N_{F}}^{(0)}\right\}$ is the desired data code. To take advantage of time domain orthogonality, time domain despreading is carried out firstly, with output

$$
\begin{aligned}
r_{0}(\bar{m}) & =\frac{1}{N_{T}} \sum_{\hat{i}=0}^{N_{T}-1} r(\bar{m}, \bar{i})\left(c_{N_{T}, \bar{i}}^{(1)} s_{\bar{m}, \bar{i}}\right) \\
& =S_{T, 0}(\bar{m})+I_{T, 0}(\bar{m})+N_{T, 0}(\bar{m})
\end{aligned}
$$

where $S_{T, 0}(\bar{m})=\sqrt{P} h_{\bar{m}} d_{0} c_{N_{F}, \bar{m}}^{(0)}$ is the useful signal component and $I_{T, 0}(\bar{m})$ is the interference term from the $K_{C}$ code channels in $\Omega_{F}$, which employ the same time domain spreading code as, but different frequency domain spreading code from the desired data channel. The $K_{C}$ interference codes in $\Omega_{F}$ can be expressed as $k=k_{F} N_{T}, k_{F}=1,2, \ldots, K_{C}$. Then $I_{T, 0}(\bar{m})$ is given by

$$
I_{T, 0}(\bar{m})=\sqrt{P} h_{\bar{m}} \sum_{k_{F}=1}^{K_{C}} d_{k_{F} N_{T}} c_{N_{F}, \bar{m}}^{\left(k_{F}\right)} .
$$

Finally, $N_{T, 0}(\bar{m})$ is the background noise with variance

$$
\sigma_{N_{T, 0}}^{2}=\frac{\sigma_{n}^{2}}{N_{T}}=\frac{N_{0}}{N_{T} T_{e}} .
$$




\section{Pure MMSE Detection}

At the zeroth stage (or original stage) of MCI cancellation, the regenerated MCI is unavailable. Thus pure MMSE detection is used to combine the useful signals from different sub-carriers. As well-known, the weights of MMSE are given by [9]

$$
\begin{aligned}
\omega(\bar{m}) & =\frac{E\left\{\sqrt{P} d_{0} r_{0}^{*}(\bar{m}) \mid \hat{h}_{\bar{m}}\right\} \cdot c_{N_{F}, \bar{m}}^{(0)}}{E\left\{\left|r_{0}(\bar{m})\right|^{2} \mid \hat{h}_{\bar{m}}\right\}} \\
& =\frac{P \hat{h}_{\bar{m}}^{*}}{E\left\{\left|S_{T, 0}(\bar{m})\right|^{2} \mid \hat{h}_{\bar{m}}\right\}+E\left\{\left|I_{T, 0}(\bar{m})\right|^{2} \mid \hat{h}_{\bar{m}}\right\}+\sigma_{T, n}^{2}}
\end{aligned}
$$

where $\hat{h}_{\bar{m}}$ is given by (18), $E\left\{\left|S_{T, 0}(\bar{m})\right|^{2} \mid \hat{h}_{\bar{m}}\right\}$ and $E\left\{\left|I_{T, 0}(\bar{m})\right|^{2} \mid \hat{h}_{\bar{m}}\right\}$ are the conditional power (or variance) of $S_{T, 0}(\bar{m})$ and $I_{T, 0}(\bar{m})$, respectively. Note that (23) is obtained with the assumption of full load. However, it is considered in this paper as an approximation of MMSE weight when the system is not full loaded (i.e., $K_{C}<N_{F}-1$ ). Therefore, the weights of pure MMSE detection are given by

$$
\begin{aligned}
\omega(\bar{m}) & =\frac{\hat{h}_{\bar{m}}^{*}}{\left(1+K_{C}\right)\left|\hat{h}_{\bar{m}}\right|^{2}+\frac{\sigma_{T, n}^{2}}{P}} \\
& =\frac{\hat{h}_{\bar{m}}^{*}}{\left(1+K_{C}\right)\left|\hat{h}_{\bar{m}}\right|^{2}+\left(\frac{N_{T} E_{s}}{N_{0}}\right)^{-1}}
\end{aligned}
$$

where $\left(E_{s}\right) /\left(N_{0}\right)=\left(P T_{e}\right) /\left(N_{0}\right)$.

Consider the $N_{F}$ sub-carriers employed by the zeroth symbol, which can be denoted by $\bar{m}=m N_{B}$, where $m=0,1, \ldots,\left(N_{F}-1\right)$. The output of the frequency domain despreader is given by

$$
\begin{aligned}
y_{0} & =\sum_{m=0}^{N_{F}-1} r_{0}\left(m N_{B}\right)\left[c_{N_{F}, m}^{(0)} \omega\left(m N_{B}\right)\right] \\
& =S_{0}+\mathrm{MCI}_{0}+\eta_{0}
\end{aligned}
$$

where $S_{0}$ is the desired component, given by

$$
S_{0}=\sqrt{P} d_{0}\left(\sum_{m=0}^{N_{F}-1} h_{m N_{B}} \omega\left(m N_{B}\right)\right) .
$$

$\mathrm{MCI}_{0}$ is the MCI generated by code channels from $\Omega_{F}$. Since the data on the zeroth code, $d_{0}$, is the desired one, the $K_{C}$ interference data in $\Omega_{F}$ are denoted by $d_{k_{F} N_{T}}, k_{F}=1, \ldots, K_{C}$. Thus, $\mathrm{MCI}_{0}$ is given by

$$
\mathrm{MCI}_{0}=\sum_{m=0}^{N_{F}-1} I_{T, 0}\left(m N_{B}\right)\left[c_{N_{F}, m}^{(0)} \omega\left(m N_{B}\right)\right]
$$

and $\eta_{0}$ is the background noise, given by

$$
\eta_{0}=\sum_{m=0}^{N_{F}-1} N_{T, 0}\left(m N_{B}\right)\left[c_{N_{F}, m}^{(0)} \omega\left(m N_{B}\right)\right] .
$$

Therefore, the decision variable is given by

$$
z_{0}=\frac{1}{\sqrt{P}} \cdot \frac{y_{0}}{\sum_{m=0}^{N_{F}-1} \hat{h}_{m N_{B}} \omega\left(m N_{B}\right)} .
$$

With hard decision, the data on the zeroth code can be recovered by the minimum distance criteria

$$
\hat{d}_{0}=\arg \left\{\min _{\left\{s_{0}, s_{1}, s_{2}, s_{3}\right\}}\left|z_{0}-s_{i}\right|^{2}\right\}
$$

where $s_{0}=(1+j) / \sqrt{2}, s_{1}=(-1+j) / \sqrt{2}, s_{2}=(-1-$ $j) / \sqrt{2}$, and $s_{3}=(1-j) / \sqrt{2}$ are the points in the QPSK signal constellation.

\section{Hybrid MCI Cancellation and MMSE Detection}

With tentative data decisions $\hat{d}_{k_{F} N_{T}}^{(s-1)}, k_{F}=1,2, \ldots, K_{C}$, of the $(s-1)$ th stage, MCI cancellation could be carried out in the $s$ th stage. With estimated channel fading $\hat{h}_{m N_{B}}$, MCI in the $s$ th stage can be regenerated for the $\left(m N_{B}\right)$ th sub-carrier of the zeroth code channel as follows

$$
\hat{I}_{T, 0}^{(s)}\left(m N_{B}\right)=\sqrt{P} \sum_{k_{F}=1}^{K_{C}} \hat{d}_{k_{F} N_{T}}^{(s-1)} \hat{h}_{m N_{B}} c_{N_{F}, m}^{\left(k_{F}\right)}
$$

It can be seen that the accuracy of the regenerated interference relies on the reliability of data decisions on the previous stage and channel estimations. The signal after MCI cancellation is given by

$$
\begin{aligned}
r_{0}^{(s)}\left(m N_{B}\right) & =r_{0}\left(m N_{B}\right)-\hat{I}_{T, 0}^{(s)}\left(m N_{B}\right) \\
& =S_{T, 0}\left(m N_{B}\right)+I_{T, 0}^{(s)}\left(m N_{B}\right)+N_{T, 0}\left(m N_{B}\right)
\end{aligned}
$$

where $I_{T, 0}^{(s)}\left(m N_{B}\right)$ is the residual MCI after $s$ th stage of cancellation, given by

$$
\begin{aligned}
& I_{T, 0}^{(s)}\left(m N_{B}\right)= I_{T, 0}\left(m N_{B}\right)-\hat{I}_{T, 0}^{(s)}\left(m N_{B}\right) \\
&=\sqrt{P} \sum_{k_{F}=1}^{K_{C}}\left(d_{k_{F} N_{T}} h_{m N_{B}}\right. \\
&\left.\quad-\hat{d}_{k_{F} N_{T}}^{(s-1)} \hat{h}_{m N_{B}}\right) c_{N_{F}, m}^{\left(k_{F}\right)}
\end{aligned}
$$

The variance of the residual MCI on $s$ th stage is dependent on the data decision error $d_{k_{F} N_{T}}-\hat{d}_{k_{F} N_{T}}^{(s-1)}$ and the channel estimation error $e_{P, m N_{B}}=h_{m N_{B}}-\hat{h}_{m N_{B}}$. These two errors are somewhat correlated. However, for precise channel estimation with small $e_{P, m N_{B}}$, this correlation should be small enough and so is neglected. Given $\hat{h}_{m N_{B}}$, the variance of the residual MCI on $s$ th stage is approximated by

$$
\begin{aligned}
\sigma_{I_{T, 0}}^{2}(s)= & P \sum_{k_{F}=1}^{K_{C}} E\left\{\left|d_{k_{F} N_{T}} h_{m N_{B}}-\hat{d}_{k_{F} N_{T}}^{(s-1)} \hat{h}_{m N_{B}}\right|^{2}\right\} \\
\approx & P \sum_{k_{F}=1}^{K_{C}}\left[E\left\{\left|\left(d_{k_{F} N_{T}}-\hat{d}_{k_{F} N_{T}}^{(s-1)}\right) h_{m N_{B}}\right|^{2}\right\}\right. \\
& \left.+E\left\{\left|d_{k_{F} N_{T}}\left(h_{m N_{B}}-\hat{h}_{m N_{B}}\right)\right|^{2}\right\}\right] .
\end{aligned}
$$


Defining $P_{b}^{(s-1)}$ as the BER of the $(s-1)$ th stage and assuming $d_{0}=s_{0}$, when Gray mapping is employed, $E\left\{\left|d_{k_{F} N_{T}}-\hat{d}_{k_{F} N_{T}}^{(s-1)}\right|^{2}\right\}$ is given by

$$
\begin{aligned}
E\{\mid & \left.d_{k_{F} N_{T}}-\left.\hat{d}_{k_{F} N_{T}}^{(s-1)}\right|^{2}\right\} \\
= & \left(1-P_{b}^{(s-1)}\right)^{2}\left|s_{0}-s_{0}\right|^{2} \\
& +\left(1-P_{b}^{(s-1)}\right) P_{b}^{(s-1)}\left|s_{0}-s_{1}\right|^{2} \\
& +\left(P_{b}^{(s-1)}\right)^{2}\left|s_{0}-s_{2}\right|^{2} \\
& +\left(1-P_{b}^{(s-1)}\right) P_{b}^{(s-1)}\left|s_{0}-s_{3}\right|^{2} \\
= & 4 P_{b}^{(s-1)} .
\end{aligned}
$$

Thus

$$
\sigma_{I_{T, 0}}^{2}(s)=4 K_{C} P \cdot P_{b}^{(s-1)}\left|h_{m N_{B}}\right|^{2}+\frac{K_{C} N_{0}}{\gamma N_{T} T_{e} \beta} .
$$

The new weights of MMSE with MCI cancellation can be expressed as (37), shown at the bottom of the page, which shows the formula of MMSE weights for analytical purpose and is employed in next section for performance evaluation. However, in practice, the original formula of MMSE weights, i.e., (23), should be used for weight estimation. According to (23), since the power $P$ and channel fading $\hat{h}_{\bar{m}}$ can be estimated, without loss of ergodicity, the MMSE weight can be obtained by estimating the ensemble average $E\left\{\left|r_{0}^{(s)}(\bar{m})\right|^{2} \mid \hat{h}_{\bar{m}}\right\}$ with time and code average, i.e.,

$$
E\left\{\left|r_{0}^{(s)}(\bar{m})\right|^{2} \mid \hat{h}_{\bar{m}}\right\} \approx \frac{1}{K \gamma} \sum_{k=0}^{K-1} \sum_{i^{\prime}=0}^{\gamma-1}\left|r_{k, i^{\prime}}^{(s)}(\bar{m})\right|^{2}
$$

where $r_{k, i^{\prime}}^{(s)}(\bar{m})$ is the output from the time domain despreader for the $\left(i^{\prime} N_{T}\right)$ th to $\left[\left(i^{\prime}+1\right) N_{T}-1\right]$ th OFCDM symbols on the $\bar{m}$ th sub-carrier of the $k$ th code.

Finally, the decision variable for the data signal on the zeroth code channel after hybrid MCI cancellation and MMSE detection is expressed as

$$
\begin{aligned}
y_{0}^{(s)} & =\sum_{m=0}^{N_{F}-1} r_{0}^{(s)}\left(m N_{B}\right)\left[c_{N_{F}, m}^{(0)} \omega^{(s)}\left(m N_{B}\right)\right] \\
& =S_{0}^{(s)}+\mathrm{MCI}_{0}^{(s)}+\eta_{0}^{(s)}
\end{aligned}
$$

where $S_{0}^{(s)}$ is the desired signal component contributed from the $N_{T}$ OFCDM symbols in time domain over $N_{F}$ sub-carriers, given by

$$
S_{0}^{(s)}=\sqrt{P} d_{0}\left(\sum_{m=0}^{N_{F}-1} h_{m N_{B}} \omega^{(s)}\left(m N_{B}\right)\right) .
$$

$\mathrm{MCI}_{0}^{(s)}$ is the residual MCI contributed from channel estimation error and data decision error of other $K_{C}$ interfering code channels, given by

$$
\mathrm{MCI}_{0}^{(s)}=\sum_{m=0}^{N_{F}-1} I_{T, 0}^{(s)}\left(m N_{B}\right)\left[c_{N_{F}, m}^{(0)} \omega^{(s)}\left(m N_{B}\right)\right]
$$

and $\eta_{0}^{(s)}$ is the background noise component, given by

$$
\eta_{0}^{(s)}=\sum_{m=0}^{N_{F}-1} N_{T, 0}\left(m N_{B}\right)\left[c_{N_{F}, m}^{(0)} \omega^{(s)}\left(m N_{B}\right)\right] .
$$

Note that with hybrid detection, not only MCI but also useful signal and background noise vary with stages because of MMSE weight updating.

\section{Performance Evaluation}

Conditioned on the real and estimated fading factors on $N_{F}$ sub-carriers $\left\{h_{m N_{B}}, \hat{h}_{m N_{B}}\right\}$, the decision variable, $y_{0}^{(s)}$, at the $s$ th stage of cancellation is given by (39) with the desired component, $S_{0}^{(s)}$, the residual MCI, $\mathrm{MCI}_{0}^{(s)}$, and the noise term, $\eta_{0}^{(s)}$ given by (40), (41), and (42), respectively. The complex data contained in (40), (41), and (33) can be written as

$$
d_{k_{F} N_{T}}=\left(d_{I, k_{F} N_{T}}+j \cdot d_{Q, k_{F} N_{T}}\right) / \sqrt{2}
$$

where $d_{I, k_{F} N_{T}}$ and $d_{Q, k_{F} N_{T}}$ are real and take 1 or -1 with equal probability. The tentative decisions $\hat{d}_{k_{F} N_{T}}^{(s)}$ can be written in a similar way. Defining the data error vector as

$$
e_{k_{F} N_{T}}^{(s-1)}=d_{k_{F} N_{T}}-\hat{d}_{k_{F} N_{T}}^{(s-1)}=e_{I, k_{F} N_{T}}^{(s-1)}+j \cdot e_{Q, k_{F} N_{T}}^{(s-1)}
$$

the error probabilities of the real and imaginary parts are supposed to be equal and given by

$$
P\left(e_{I, k_{F} N_{T}}^{(s-1)}\right)= \begin{cases}1-P_{b}^{(s-1)}, & e_{I, k_{F} N_{T}}^{(s-1)}=0 \\ P_{b}^{(s-1)}, & e_{I, k_{F} N_{T}}^{(s-1)} \neq 0 .\end{cases}
$$

Given the value of $\left\{h_{m N_{B}}, \hat{h}_{m N_{B}}, m=0, \ldots, N_{F}-\right.$ $1\},\left\{d_{k_{F} N_{T}}, k_{F}=1, \ldots, K_{C}\right\}$, and $\left\{e_{k_{F} N_{T}}^{(s-1)}, k_{F}=\right.$ $\left.1, \ldots, K_{C}\right\}$, the value of $\mathrm{MCI}_{0}^{(s)}$ in (41) can be determined. In order to derive the average error probability, the following definitions of the conditions are introduced for averaging conditional error probabilities. $\mathbf{h}$ is defined as a vector of the real and estimated $N_{F}$ channel fading factors, i.e.,

$$
\mathbf{h}=\left\{h_{0}, h_{N_{B}}, \ldots, h_{\left(N_{F}-1\right) N_{B}}, \hat{h}_{0}, \hat{h}_{N_{B}}, \ldots, \hat{h}_{\left(N_{F}-1\right) N_{B}}\right\} \text {. }
$$

$$
\omega^{(s)}\left(m N_{B}\right)=\frac{P \hat{h}_{m N_{B}}^{*}}{P\left|\hat{h}_{m N_{B}}\right|^{2}+\sigma_{I_{T, 0}}^{2}(s)+\sigma_{N_{T, 0}}^{2}}=\frac{\hat{h}_{m N_{B}}^{*}}{\left(1+4 P_{b}^{(s-1)} K_{C}\right)\left|\hat{h}_{m N_{B}}\right|^{2}+\left(\frac{\gamma N_{T} \beta}{K_{C}} \cdot \frac{E_{s}}{N_{0}}\right)^{-1}+\left(\frac{N_{T} E_{s}}{N_{0}}\right)^{-1}}
$$


d is defined as a vector of the $K_{C}$ interference channel data, i.e.,

$$
\mathbf{d}=\left\{d_{N_{T}}, d_{2 N_{T}}, \ldots, d_{K_{C} N_{T}}\right\} .
$$

Since each element in $\mathbf{d}$ has four possible values, the total number of different sequences in $\mathbf{d}$ is $4^{K_{C}}$. Thus, a new data vector $\mathbf{d}(j)$ is defined as the $j$ th $\left(j=0,1, \ldots, 4^{K_{C}}-1\right)$ sequence of $\mathbf{d}$.

$\mathbf{e}_{\mathrm{I}}$ is defined as a vector of the real parts of the $K_{C}$ data errors

$$
\mathbf{e}_{\mathrm{I}}=\left\{e_{I, N_{T}}^{(s-1)}, e_{I, 2 N_{T}}^{(s-1)}, \ldots, e_{I, K_{C} N_{T}}^{(s-1)}\right\}
$$

Assuming that there are $n_{I}$ bit errors out of the $K_{C}$ elements in $\mathbf{e}_{\mathrm{I}}$, the number of different error sequences is

$$
\left(\begin{array}{c}
K_{C} \\
n_{I}
\end{array}\right)=\frac{K_{C} !}{n_{I} !\left(K_{C}-n_{I}\right) !}
$$

Thus, $\mathbf{e}_{\mathrm{I}}\left(n_{I}, i_{I}\right)$ is defined as the $i_{I}$ th

$$
\left(i_{I}=0,1, \ldots,\left(\begin{array}{c}
K_{C} \\
n_{I}
\end{array}\right)-1\right)
$$

error sequence. Similarly, $\mathbf{e}_{\mathrm{Q}}\left(n_{Q}, i_{Q}\right)$ is defined as the $i_{Q}$ th error sequence of $\mathbf{e}_{\mathrm{Q}}$, a vector of the imaginary parts of the $K_{C}$ data errors

$$
\mathbf{e}_{\mathrm{Q}}=\left\{e_{Q, N_{T}}^{(s-1)}, e_{Q, 2 N_{T}}^{(s-1)}, \ldots, e_{Q, K_{C} N_{T}}^{(s-1)}\right\} .
$$

Since the error probabilities of real and imaginary parts are equal, the conditional error probability at the $s$ th stage is given by

$$
\begin{aligned}
P_{b}^{(s)} & \left(\hat{d}_{0}^{(s)} \neq d_{0} \mid \mathbf{h}, \mathbf{d}(j), \mathbf{e}_{\mathrm{I}}\left(n_{I}, i_{I}\right), \mathbf{e}_{\mathrm{Q}}\left(n_{Q}, i_{Q}\right)\right) \\
= & \frac{1}{2} \operatorname{Pr}\left(\operatorname{Re}\left(S_{0}^{(s)}+\mathrm{MCI}_{0}^{(s)}+\eta_{0}^{(s)}\right) \leq\left. 0\right|_{d_{I, 0}=d_{Q, 0}=1}\right) \\
& +\frac{1}{2} \operatorname{Pr}\left(\operatorname{Re}\left(S_{0}^{(s)}+\mathrm{MCI}_{0}^{(s)}+\eta_{0}^{(s)}\right) \leq\left. 0\right|_{d_{I, 0}=1, d_{Q, 0}=-1}\right) \\
= & \frac{1}{2} Q\left(\left.\frac{\operatorname{Re}\left(S_{0}^{(s)}+\mathrm{MCI}_{0}^{(s)}\right)}{\sigma_{\eta}(s) / \sqrt{2}}\right|_{d_{I, 0}=d_{Q, 0}=1}\right) \\
& +\frac{1}{2} Q\left(\left.\frac{\operatorname{Re}\left(S_{0}^{(s)}+\mathrm{MCI}_{0}^{(s)}\right)}{\sigma_{\eta}(s) / \sqrt{2}}\right|_{d_{I, 0}=1, d_{Q, 0}=-1}\right)
\end{aligned}
$$

where $Q(z)=(1 / \sqrt{2 \pi}) \int_{z}^{+\infty} e^{-\left(t^{2} / 2\right)} d t$ for $z \geq 0, \sigma_{\eta}^{2}(s)$ stands for the variance of $\eta_{0}^{(s)}$, and the factor of $\sqrt{2}$ is introduced because only the real part of the Gaussian noise is considered with the variance equal to half of that of the complex Gaussian noise.

In order to obtain the average bit error rate, (50) must be averaged over all conditions. Suppose that the effects of the four conditions, $\mathbf{h}, \mathbf{d}(j), \mathbf{e}_{\mathrm{I}}\left(n_{I}, i_{I}\right)$, and $\mathbf{e}_{\mathrm{Q}}\left(n_{Q}, i_{Q}\right)$, on the BER performance are independent to each other. Equation (51) can be averaged over these conditions one by one. Let's average (50) over $\mathbf{e}_{\mathrm{I}}\left(n_{I}, i_{I}\right)$ first. For a certain data error vector, not only the numbers of bit errors but also the positions of bit errors affect the value of $\mathrm{MCI}_{0}^{(s)}$. The probability of the $i_{I}$ th order error sequence with $n_{I}$ bit errors out of $K_{C}$ codes is given by

$$
P\left(n_{I}\right)=\left(P_{b}^{(s-1)}\right)^{n_{I}}\left(1-P_{b}^{(s-1)}\right)^{K_{C}-n_{I}}
$$

where the decision errors on $K_{C}$ interference codes are supposed to be independent. When $n_{I}$ is large, (51) is very small. Thus, in numerical calculation, only a few small values of $n_{I}$ need to be considered. Therefore, the average of (50) over $\mathbf{e}_{\mathrm{I}}\left(n_{I}, i_{I}\right)$ is given by

$$
\begin{aligned}
P_{b}^{(s)} & \left(\hat{d}_{0}^{(s)} \neq d_{0} \mid \mathbf{h}, \mathbf{d}(j), \mathbf{e}_{\mathrm{Q}}\left(n_{Q}, i_{Q}\right)\right) \\
= & \sum_{n_{I}=0}^{K_{C}} P\left(n_{I}\right) \sum_{i_{I}=0}^{K_{C}} n_{I}-1 \\
& \left.\mathbf{e}_{\mathrm{I}}\left(n_{I}, i_{I}\right), \mathbf{e}_{\mathrm{Q}}\left(n_{Q}, i_{Q}\right)\right)
\end{aligned}
$$

Similarly, the average of (52) over $\mathbf{e}_{\mathrm{Q}}\left(n_{Q}, i_{Q}\right)$ is given by

$$
\begin{aligned}
P_{b}^{(s)} & \left(\hat{d}_{0}^{(s)} \neq d_{0} \mid \mathbf{h}, \mathbf{d}(j)\right)=\sum_{n_{Q}=0}^{K_{C}} P(n) \\
& \left(\begin{array}{c}
K_{C} \\
\left.n_{Q}\right)-1
\end{array}\right. \\
\times & \sum_{i_{Q}=0}^{(s)}\left(\hat{d}_{0}^{(s)} \neq d_{0} \mid \mathbf{h}, \mathbf{d}(j), \mathbf{e}_{\mathrm{Q}}\left(n_{Q}, i_{Q}\right)\right)
\end{aligned}
$$

Then (53) should be averaged over all possible $K_{C}$ interference data

$$
P_{b}^{(s)}\left(\hat{d}_{0}^{(s)} \neq d_{0} \mid \mathrm{h}\right)=\sum_{j=0}^{4^{K} C-1} P_{b}^{(s)}\left(\hat{d}_{0}^{(s)} \neq d_{0} \mid \mathbf{h}, \mathbf{d}(j)\right)
$$

In the special case (i.e., the zeroth stage or pure MMSE detection), regenerated MCI or the error vector does not exist. Thus, the error vectors in (50) should be removed.

Finally, $P_{b}^{(s)}\left(\hat{d}_{0}^{(s)} \neq d_{0} \mid \mathbf{h}\right)$ is averaged over all $\mathbf{h}$, which can be numerically evaluated by a Monte Carlo approach [16].

\section{NUMERICAL RESULTS}

In this section, some representative numerical results are presented. It is assumed that the system bandwidth is $100 \mathrm{MHz}$. The number of sub-carriers $M$ is equal to 1024 . The average SNR per bit is defined as $\operatorname{SNR}_{b}=((P+\beta P / K) N) /\left(2 \sigma_{n}^{2}\right)=$ $\left(N E_{s}\right) /\left(2 N_{0}\right)(1+\beta / K)$ and the system load is measured as $K / N$. Unless noted otherwise, $K / N$ is set to 0.75 . The parameter $\gamma$ of determining the channel estimation window size $\gamma N_{T}$ is set to be $\gamma=3$. The coherence bandwidth $\Delta f_{c}$ is set to $1 \mathrm{MHz}$ 


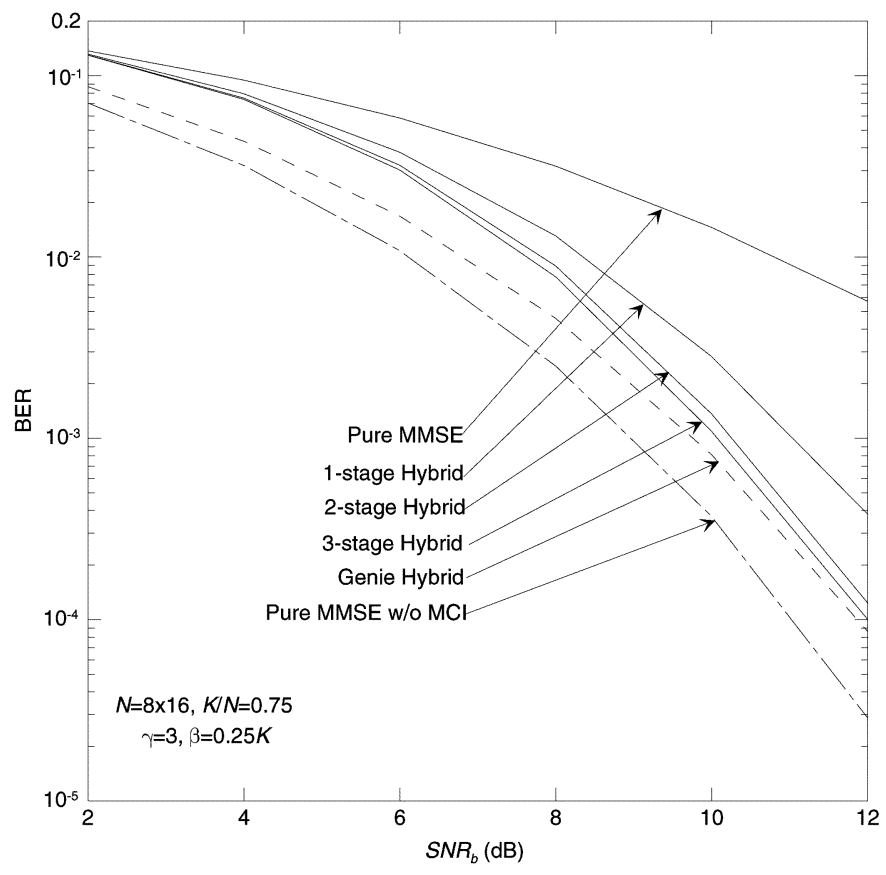

Fig. 4. Performance of hybrid MCI cancellation and MMSE detection with $N=8 \times 16$.

[17], so the correlation coefficient between two adjacent subcarriers of interest is given by $\rho_{0, N_{B}}=1 / \sqrt{1+\left(100 / N_{F}\right)^{2}}$ (see (12)). The correlated Rayleigh fading channels are generated according to [18].

First of all, the BER performance of hybrid MCI cancellation and MMSE detection is shown in Fig. 4 for $N=N_{T} \times N_{F}=$ $8 \times 16$ and $\rho_{0, N_{B}} \approx 0.16$. The power ratio $\beta$ is set to $0.25 K$. A 3 -stage MCI cancellor, including zeroth to third stage, is considered when the system load is $K / N=0.75$. The system performance of genie hybrid detection (assuming perfect tentative decisions) is shown in the figure for comparison. Note that in genie hybrid, possible data errors are caused by imperfect channel estimation and background noise. Moreover, the performance of pure MMSE without MCI (i.e., all data code channels are assumed to be orthogonal) is also shown. It can be seen from Fig. 4 that the hybrid detection performs much better than pure MMSE when $\mathrm{SNR}_{b}$ is large. The BER decreases as the number of stages increases. The performance improvement between the pure MMSE and the hybrid detection with the first stage is significant. The gap in BER between the first stage and second stage is also big. However, the improvement beyond the second stage is insignificant. The room for improvement between the second stage and genie hybrid is small, especially when $\mathrm{SNR}_{b}$ is large. In conclusion, the BER performance improvement for the hybrid detection decreases as the stages increases. Considering 2-stage is sufficient.

Fig. 5 shows the BER performance as a function of system load $K / N$ for different number of stages. The $\mathrm{SNR}_{b}$ is fixed to $10 \mathrm{~dB}$ and the power ratio $\beta$ is set to $0.25 K$. It can be seen that with various system load, the hybrid detection exhibits much better performance than the pure MMSE. When the system load is light, a single stage is required. However, when the system load is heavy, considering 2-stage is necessary. This is consistent with Fig. 4.

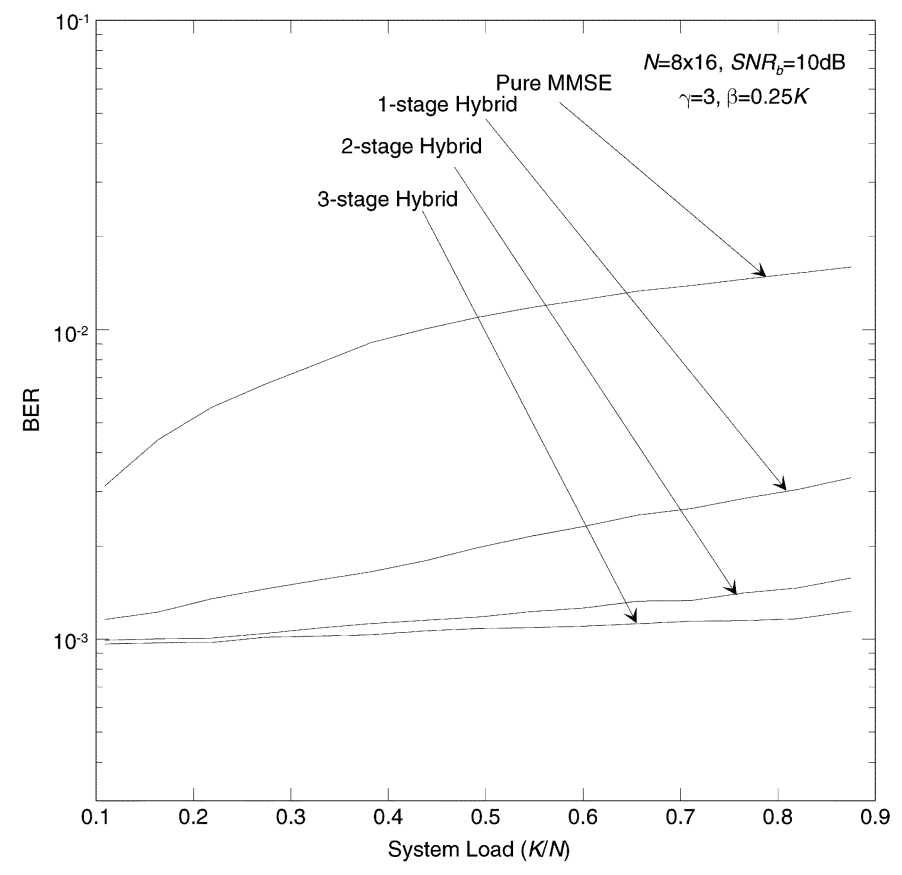

Fig. 5. System performance as a function of the system load.

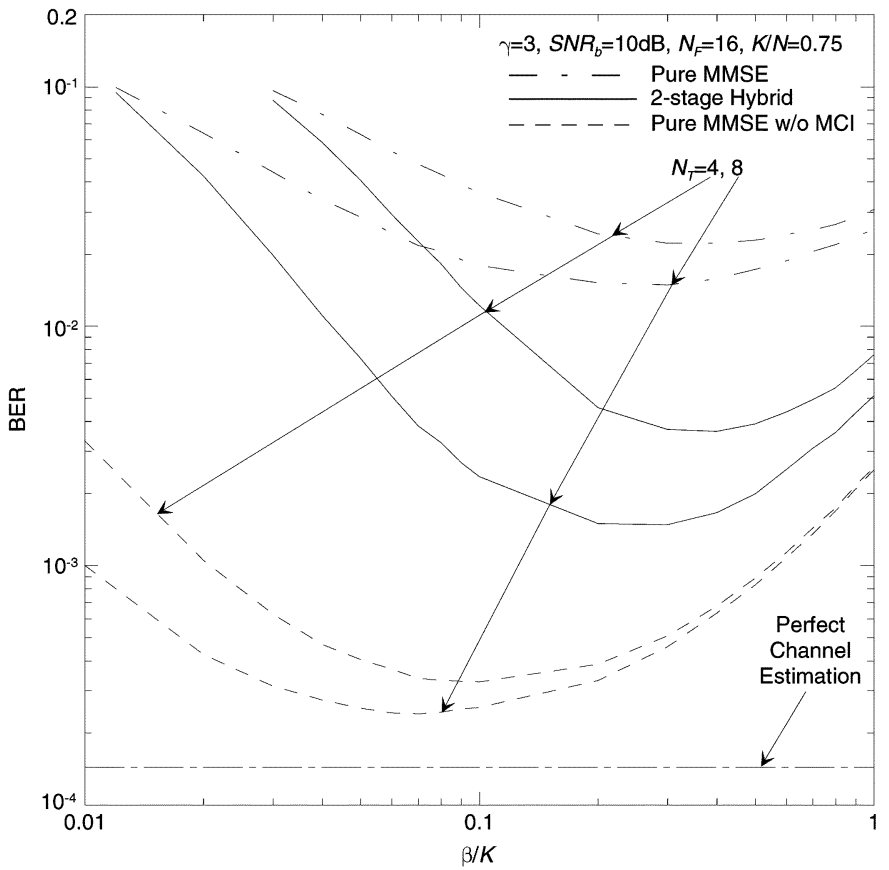

Fig. 6. Optimum power ratio between pilot and data channels.

Fig. 6 illustrates the effect of power ratio $\beta / K$ on the performance of the two-dimensionally spreading system with 2-stage hybrid detection. The frequency domain spreading factor $N_{F}$ is set to 16 and the time domain spreading factor $N_{T}$ takes 4 and 8 , respectively. Again, the correlation coefficient $\rho_{0, N_{B}} \approx 0.16$ and $\mathrm{SNR}_{b}$ is $10 \mathrm{~dB}$. The system performances of pure MMSE without MCI and of perfect channel estimation are shown in Fig. 6 as comparisons. It can be seen from the figure that for both 2-stage hybrid and pure MMSE, when $\beta / K$ is small, the pilot channel has small power and this results in poor channel estimation. Thus, the system performance degrades and the BER 


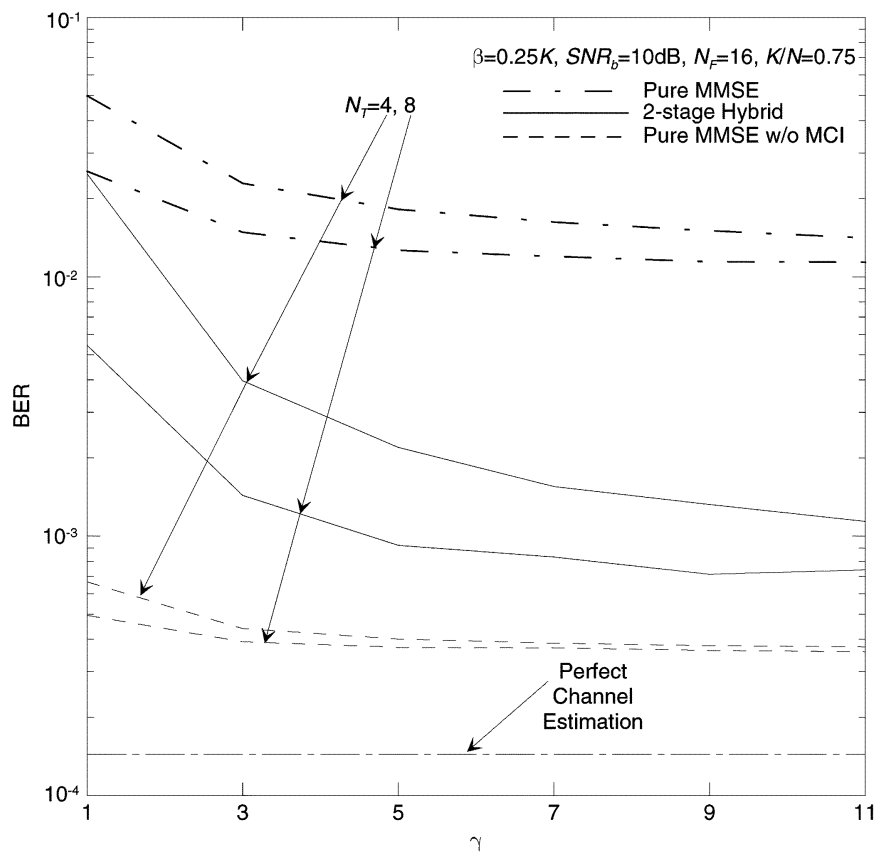

Fig. 7. Effect of $\gamma$ on the system performance.

of hybrid detection is close to that of pure MMSE. When $\beta / K$ increases, BER performance improves as the quality of channel estimation improves, and hybrid detection performs better and better than pure MMSE. BER reaches a minimum value for a particular value of $\beta / K$. Further increasing $\beta / K$ beyond that value increases BER, and the performance gap between hybrid detection and pure MMSE is also reduced. This is because there is too much power assigned to the pilot channel and small power to data channels so that data channels become vulnerable to channel noise. When $N_{T}$ increases from 4 to 8 , the optimum value of $\beta / K$ gets smaller. It is because in the slow fading channel, the energy in pilot channel increases as $N_{T}$ increases. Therefore, less portion of power is needed for the pilot channel to get the optimum system performance. In general, for hybrid detection to give much better performance than pure MMSE, the power ratio $\beta / K$ should be allocated in the range of $[0.2,0.6]$, and a near optimum power ratio, i.e., $\beta / K=0.25$, can be seen irrespective of the values of $N_{T}$. Similar trends in performance can be seen for MMSE without MCI. The only difference is that the MMSE without MCI requires much smaller value of $\beta / K$ to reach minimum error probabilities.

Fig. 7 shows the system performances of pure MMSE, 2-stage hybrid and MMSE without MCI when the parameter $\gamma$ of determining the channel estimation window size $\gamma N_{T}$ changes. Similarly, $N_{F}$ is set to 16 , and so $\rho_{0, N_{B}} \approx 0.16$. When the power ratio of pilot to total data channels is $25 \%$, i.e., $\beta / K=0.25$, the BER performance of both hybrid detection and pure MMSE improves and the performance gap between these two detections increases as $\gamma$ increases. This is because with the assumption of slow fading, the channel estimation error reduces when $\gamma$ increases. The most significant improvement is observed when $\gamma$ increases from 1 to 3 . The improvement margin reduces as $\gamma$ increases further. In conclusion, for hybrid detection to provide much better performance than pure MMSE, $\gamma=3$ is suitable

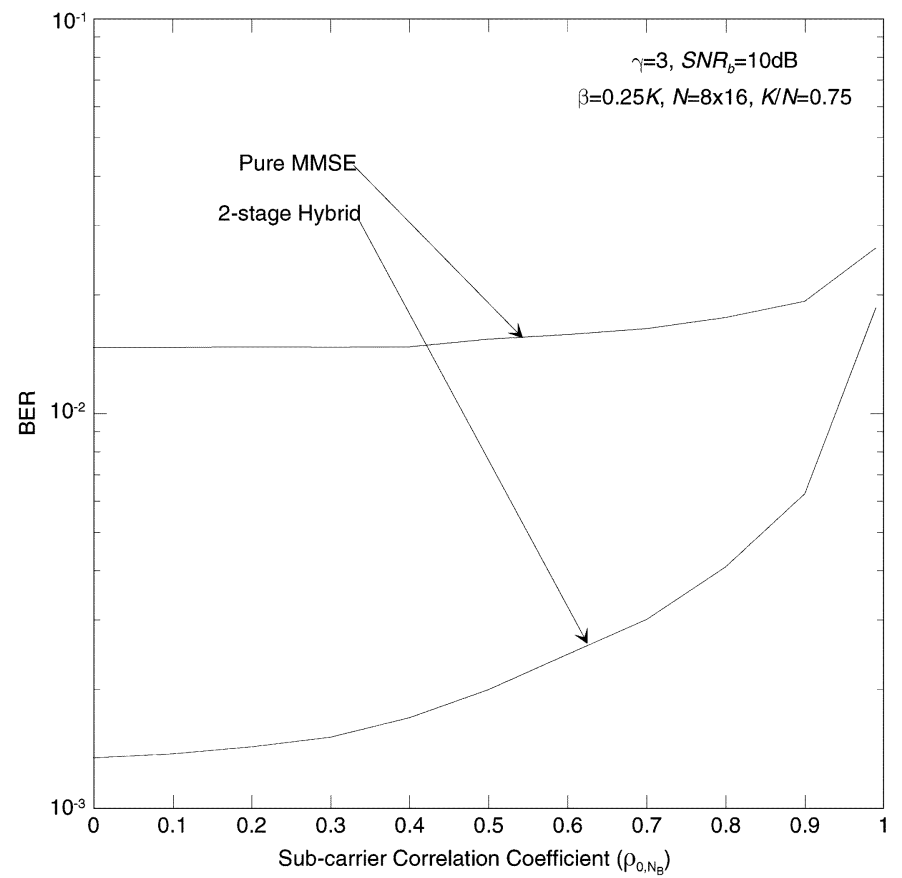

Fig. 8. Effect of sub-carrier correlation coefficient on the system performance.

for the system with $N_{T}=8$ while $\gamma=5$ is more desirable for the system with $N_{T}=4$. For MMSE without MCI, the performance is very flat although initial increase of $\gamma$ improves performance slightly.

Fig. 8 illustrates the effect of sub-carrier correlation on the BER performance with pure MMSE and 2-stage hybrid detection. Given a system bandwidth, the number of sub-carriers $M$ and spreading scheme $N=N_{T} \times N_{F}$, the correlation coefficient $\rho_{0, N_{B}}$ varies with channel coherence bandwidth $\Delta f_{c}$. It can be seen that as long as $\rho_{0, N_{B}} \leq 0.5$, the degradation of system performance is negligible. For pure MMSE, the degradation is not obvious, whereas for hybrid detection, the effect of sub-carrier correlation is more obvious when $N_{F}$ is large. Although the performance of hybrid detection is degraded by a large correlation, there is still significant improvement from hybrid detection as long as $\rho_{0, N_{B}} \leq 0.7$.

Fig. 9 illustrates the BER performance as a function of $\mathrm{SNR}_{b}$ with the hybrid 2-stage MCI cancellation and MMSE detection for various values of the frequency domain spreading factor, $N_{F}$. The time domain spreading factor, $N_{T}$, is set to 8. Different values of the sub-carrier correlation coefficient can be derived from different values of $N_{F}$. For $N_{F}=1$, it is not necessary to consider sub-carrier correlation coefficient. $\rho_{0, N_{B}}$ is 0.02 and 0.79 for $N_{F}=2$ and 128 , respectively. Thus, $\rho_{0, N_{B}}$ takes values in the range from 0.02 to 0.79 . It can be seen that the BER of the hybrid detection decreases initially as $N_{F}$ increases, especially for large $\mathrm{SNR}_{b}$. This is because more frequency diversity gain is obtained when $N_{F}$ increases. Although more MCI is caused when $N_{F}$ gets larger, MCI cancellation can suppress the MCI much. In this case, frequency diversity can still provide more gain when $N_{F}$ becomes larger. However, the performance improvement gets smaller as $N_{F}$ increases further, and finally, when $N_{F}$ is larger than 32 , the frequency diversity gain is saturated and the improvement becomes insignificant. 


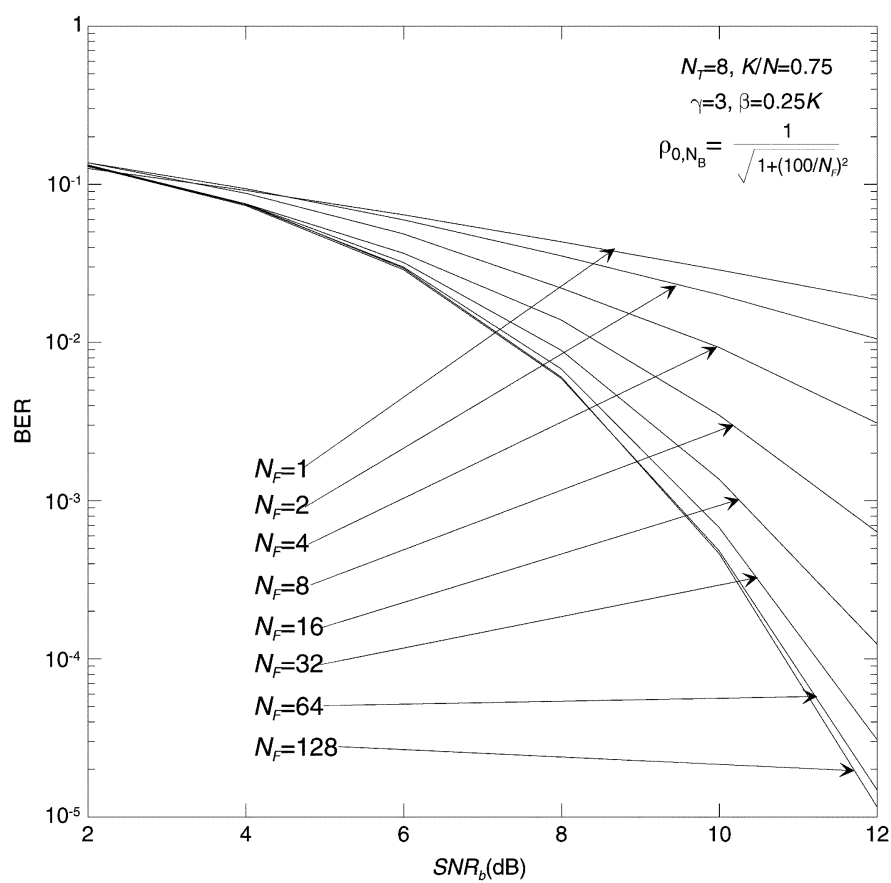

Fig. 9. System performance of 2-stage hybrid detection when $N_{F}$ changes.

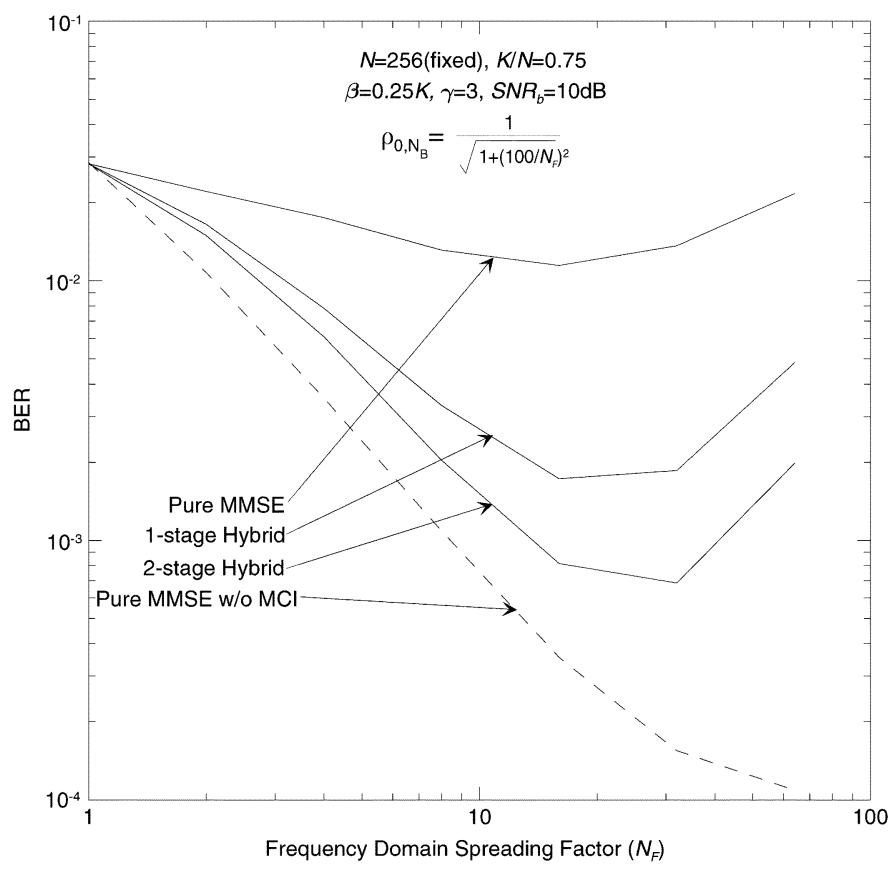

Fig. 10. System performance as a function of $N_{F}$ for a given $N$.

Fig. 10 illustrates the system performances as a function of $N_{F}$ when $N$ is fixed to 256. For both pure MMSE and hybrid detection, it can be seen from Fig. 10 that when $N_{F}$ increases from 1, the BER decreases at first and then reaches a minimum at certain $N_{F}$. The optimum $N_{F}$ is about 16 or 32 . Increasing $N_{F}$ beyond that value will degrade the system performance. This is because, in a system with fixed $N$, when $N_{F}$ increases, the frequency diversity gain increases, while MCI increases also. On the other hand, using the proposed sliding window channel estimation algorithm, the estimation quality is degraded due to decreasing $N_{T}$. When $N_{F}$ increases at first from a small

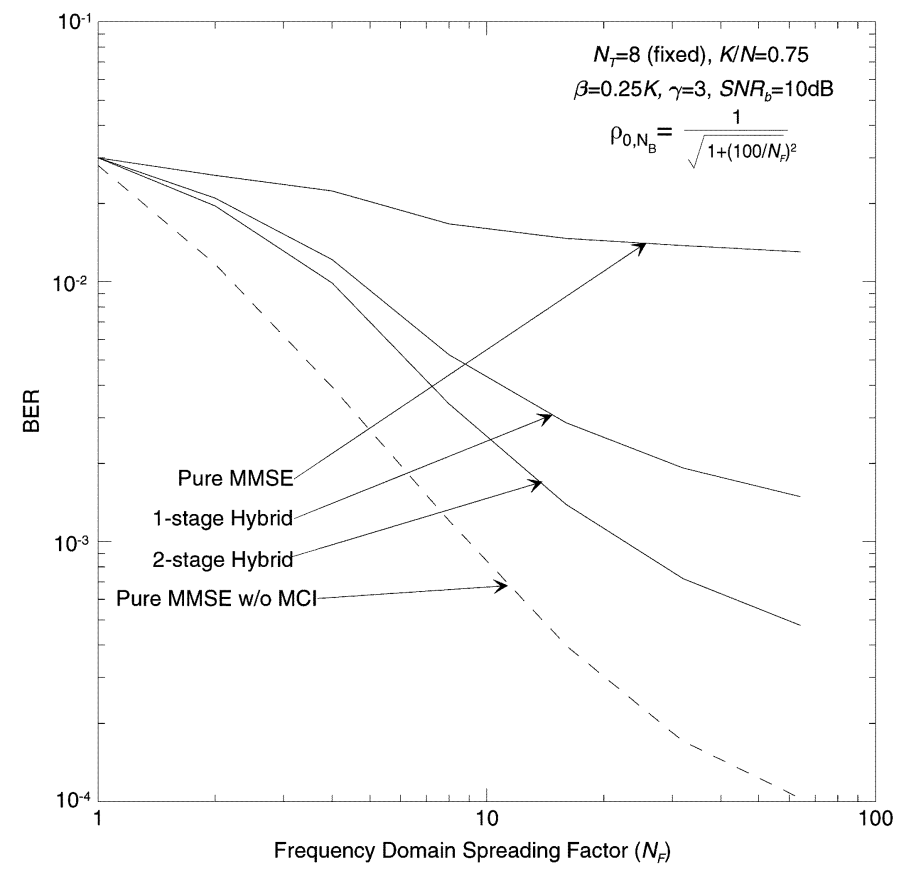

Fig. 11. System performance as a function of $N_{F}$ for a given $N_{T}$.

value, the frequency diversity gain overcomes the degradation caused by increased MCI and higher channel estimation error, and the BER reduces. However, when $N_{F}$ increases further, the degradation resulted from channel estimation error and MCI is larger than the frequency diversity gain. Therefore, the BER is increased.

Compared to Fig. 10, different performance trends can be seen from Fig. 11 where $N_{T}$ is fixed. Although the performance improvement is significant when $N_{F}$ increases initially, the improvement is slight when $N_{F}$ increases further for a large $N_{F}$. This is because frequency diversity gain is saturated when $N_{F}$ is larger (i.e., $N_{F} \geq 32$ ). This is in consistence with Fig. 9. It should also be noted that with various $N_{F}$, hybrid detection always outperforms pure MMSE.

\section{CONCLUSION}

The downlink performance of the broadband OFCDM systems with hybrid MCI cancellation and MMSE detection is investigated in this paper. The performance of pure MMSE and the hybrid detection has been compared extensively. The following conclusions are drawn.

1) The hybrid detection performs much better than the pure MMSE. But the BER performance improvement for the hybrid detection decreases as the stages increases. Considering 2-stage of MCI cancellation is sufficient.

2) Precise channel estimation is critical for the hybrid detection. The power ratio $\beta$ should be chosen properly to provide good performance. $\beta=0.25 K$ is a near optimum value for the two-dimensional spreading system with $N_{T}$ of 4 and 8 . On the other hand, in a slow fading channel, a large value of the channel estimation window size $\gamma N_{T}$ is expected. However, $\gamma=3$ is large enough for the system with $N_{T}=8$ while $\gamma=5$ is more desirable for the system with $N_{T}=4$. 
3) The degradation of system performance is negligible when the correlation coefficient of the adjacent sub-carriers of interest is less than 0.5 , whereas the degradation is large when the correlation is larger than 0.7 .

4) For the hybrid detection with fixed $N_{T}$, significant improvement can be obtained when the frequency domain spreading factor, $N_{F}$, increases from 1 to 32 . Beyond 32, the improvement is small. On the other hand, for fixed $N$ (i.e., $N=256$ ), using the proposed sliding window channel estimation algorithm, there is an optimum value of $N_{F}$ at which the performance reaches the best. The optimum $N_{F}$ is about 16 or 32 .

\section{REFERENCES}

[1] A. Chouly, A. Brajal, and S. Jourdan, "Orthogonal multicarrier techniques applied to direct sequence spread spectrum CDMA systems," in Proc. IEEE GLOBECOM, Nov. 1993, pp. 1723-1728.

[2] S. Hara and P. Ramjee, "Design and performance of multicarrier CDMA system in frequency-selective Rayleigh fading channels," IEEE Trans. Veh. Technol., vol. 48, no. 5, pp. 1584-1595, Sep. 1999.

[3] E. A. Sourour and M. Nakagawa, "Performance of orthogonal multicarrier CDMA in a multipath fading channel," IEEE Trans. Commun., vol. 44, no. 3, pp. 356-367, Mar. 1996.

[4] N. Maeda, Y. Kishiyama, H. Atarashi, and M. Sawahashi, "Variable spreading factor-OFCDM with two dimensional spreading that prioritizes time domain spreading for forward link broadband wireless access," in Proc. IEEE VTC2003-Spring, Apr. 2003, pp. 127-132.

[5] C. W. You and D. S. Hong, "Multi-carrier CDMA systems using time-domain and frequency-domain spreading codes," IEEE Trans. Commun., vol. 51, no. 1, pp. 17-21, Jan. 2003

[6] M. Fong, V. K. Bhargava, and Q. Wang, "Concatenated orthogonal/PN spreading scheme for cellular DS-CDMA systems with integrated traffic," in Proc. ICC'95, vol. 2, Jun. 1995, pp. 905-909.

[7] N. Yee and J. P. Linnartz, "Multi-carrier CDMA in indoor wireless radio networks," in Proc. IEEE Int. Symp. Personal, Indoor, and Mobile Radio Commun. (PIMRC'93), Sep. 1993, pp. 109-113.

[8] S. Kaiser, "On the performance of different detection techniques for OFDM-CDMA in fading channels," in Proc. IEEE GLOBECOM'95, Nov. 1995, pp. 2059-2063.

[9] N. Yee and J. P. Linnartz, "Wiener filtering of multi-carrier CDMA in Rayleigh fading channel," in Proc. IEEE PIMRC'94, vol. 4, Sep. 1994, pp. 1344-1347.

[10] J. P. Linnartz, "Performance analysis of synchronous MC-CDMA in mobile Rayleigh channel with both delay and Doppler spreads," IEEE Trans. Veh. Technol., vol. 50, no. 6, pp. 1375-1387, Nov. 2001.

[11] D. N. Kalofonos and J. G. Proakis, "Performance of the multi-stage detector for a MC-CDMA system in a Rayleigh fading channel," in Proc. IEEE GLOBECOM'96, Nov. 1996, pp. 1784-1788.

[12] W. C. Y. Lee, Mobile Communications Engineering: Theory and Applications, 2nd ed. New York: McGraw-Hill, 1997.

[13] N. Maeda, H. Atarashi, S. Abeta, and M. Sawahashi, "Pilot channel assisted MMSE combing in forward link for broadband OFCDM packet wireless access," IEICE Trans. Fundamentals, vol. E85-A, no. 7, pp. 1635-1646, Jul. 2002.

[14] J. G. Proakis, Digital Communications. New York: McGraw-Hill, 1995.

[15] M. K. Varanasi and B. Aazhang, "Multistage detection in asynchronous code-division multiple-access communications," IEEE Trans. Commun., vol. 38, no. 4, pp. 509-519, Apr. 1990.

[16] Z. Xing, "Parallel ensemble Monte Carlo for device simulation," in Workshop on High Performance Computing Activities in Singapore, Sep. 1995.

[17] H. Atarashi and M. Sawahashi, "Investigation of inter-carrier interference due to Doppler spread in OFCDM broadband packet wireless accesses," IEICE Trans. Commun. Special Issue on Software Defined Radio Technologies and Its Applications, vol. E86-B, 2002SRP-28, pp. 291-299, Jan. 2003.

[18] R. B. Ertel and J. H. Reed, "Generation of two equal power correlated Rayleigh fading envelopes," IEEE Commun. Lett., vol. 2, no. 10, pp. 276-278, Oct. 1998.

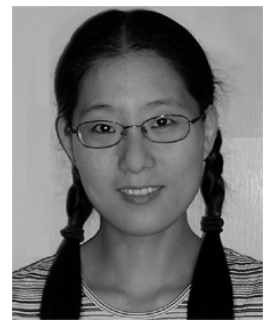

Yiqing Zhou (S'03-M'05) received the B.S. degree in communications and information engineering and the M.S. degree in signal and information processing from the Southeast University, Nanjing, China, in 1997 and 2000, respectively. In February 2004, she received the Ph.D. degree in electrical and electronic engineering from the University of Hong Kong, Hong Kong.

Since June 2004, she has been with the Department of Electrical and Electronic Engineering at the University of Hong Kong as a Postdoctoral Fellow. Her research interests include coding theory, spread spectrum, OFDM systems, interference cancellation, hybrid ARQ and other transmission techniques for wireless high speed data communications.

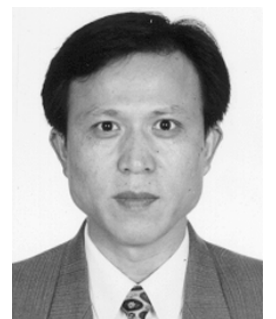

Jiangzhou Wang (M'91-SM'94) received the B.S and M.S. degrees from Xidian University, Xian, China, in 1983 and 1985, respectively, and the $\mathrm{Ph} . \mathrm{D}$. degree (with Greatest Distinction) from the University of Ghent, Gent, Belgium, in 1990, all in electrical engineering.

From 1990 to 1992, he was a Postdoctoral Fellow in the University of California at San Diego, CA, where he worked on the research and development of cellular CDMA systems. From 1992 to 1995, he was a Senior System Engineer at Rockwell International Corporation, Newport Beach, CA, where he worked on the development and system design of wireless communications. Since 1995, he has been with the University of Hong Kong, where he is currently an Associate Professor and the Coordinator of the Telecommunications Group. He has held a Visiting Professor position in NTT DoCoMo, Japan. He was a Technical Chairman of IEEE Workshop in 3G Mobile Communications, 2000. He has published over 100 papers, including more than 30 IEEE Transactions/Journal papers in the areas of wireless mobile and spread spectrum communications. He has written or edited two books, entitled Broadband Wireless Communications (Boston, MA: Kluwer, 2001) and Advances in $3 G$ Enhanced Technologies for Wireless Communications (Norwood, MA: Artech House, 2002), respectively. He holds one US patent in the GSM system.

Dr. Wang is an Editor for IEEE TRANSACTIONS ON COMMUNICATIONS and a Guest Editor for IEEE JOURNAL ON SELECTED AREAS IN COMMUNICATIONS. He is listed in Who's Who in the World.

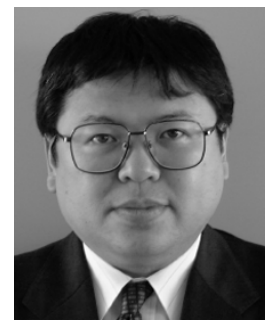

Mamoru Sawahashi (M'88) received the B.S. and M.S. degrees from Tokyo University, Tokyo, Japan, in 1983 and 1985, respectively, and the Dr.Eng. degree from the Nara Institute of Technology, Japan, in 1998.

In 1985 he joined NTT Electrical Communications Laboratories, and in 1992 he transferred to NTT Mobile Communications Network, Inc. (now NTT DoCoMo, Inc.). Since joining NTT, he has been engaged in the research of modulation/demodulation techniques for mobile radio, and research and development of wireless access technologies for W-CDMA mobile radio and broadband wireless packet access technologies for beyond IMT-2000. He is now the Director of the IP Radio Network Development Department of NTT DoCoMo, Inc.

Dr. Sawahashi is currently serving as an Editor for IEEE TRANSACTIONS ON WIRELESS COMMUNICATIONS and a Guest Editor for IEEE JOURNAL ON SELECTED AREAS IN COMMUNICATIONS. 